\title{
Performance results of the GALEX cross delay line detectors
}

Patrick N. Jelinsky, Patrick F. Morrissey, James M. Malloy, Sharon R. Jelinsky, Oswald H. W. Siegmund, et al.

Patrick N. Jelinsky, Patrick F. Morrissey, James M. Malloy, Sharon R. Jelinsky, Oswald H. W. Siegmund, Christopher Martin, David Schiminovich, Karl Forster, Ted Wyder, Peter G. Friedman, "Performance results of the GALEX cross delay line detectors," Proc. SPIE 4854, Future EUV/UV and Visible Space Astrophysics Missions and Instrumentation, (24 February 2003); doi: 10.1117/12.460013

Event: Astronomical Telescopes and Instrumentation, 2002, Waikoloa, Hawai'i, United States 


\title{
Performance Results of the GALEX cross delay line detectors
}

\author{
Patrick Jelinsky $^{\mathrm{a}}$, Patrick Morrissey ${ }^{\mathrm{b}}$, James Malloy ${ }^{\mathrm{a}}$, Sharon Jelinsky ${ }^{\mathrm{a}}$, and Oswald Siegmund ${ }^{\mathrm{a}}$, \\ Chris Martin ${ }^{\mathrm{b}}$, David Schiminovich ${ }^{\mathrm{b}}$, Karl Forster ${ }^{\mathrm{b}}$, Ted Wyder ${ }^{\mathrm{b}}$ and Peter Friedman ${ }^{\mathrm{b}}$

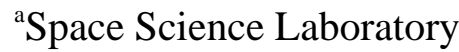 \\ University of California, Berkeley, CA 94720-7450 \\ ${ }^{\mathrm{b}}$ Space Astrophysics Laboratory \\ MS405-47, California Institute of Technology, Pasadena, CA 91125
}

\begin{abstract}
We describe the performance results for the Galaxy Evolution Explorer (GALEX) far ultraviolet (FUV) and near ultraviolet (NUV) detectors. The detectors were delivered to JPL/Caltech starting in the fall of 2000 and have undergone approximately 1000 hours of pre-flight system-level testing to date. The GALEX detectors are sealed tube microchannel plate (MCP) delay line readout detectors. They have a $65 \mathrm{~mm}$ diameter active area, which will be the largest format on orbit. The FUV detector has a spectral bandpass from 115 - $180 \mathrm{~nm}$ and the NUV detector has a bandpass from $165-300 \mathrm{~nm}$. We report here on the performance of the detectors before and after integration into the instrument. Characteristics measured include the background count rate and distribution, gain vs. applied high voltage, spatial resolution and linearity, flat fields, and quantum efficiency.
\end{abstract}

Keywords: Microchannel plates, ultraviolet detectors, GALEX, sealed tube

Correspondence: Email: patj@ssl.berkeley.edu; Telephone: 5106427399

\section{INTRODUCTION}

The Galaxy Evolution Explorer (GALEX) is a NASA imaging and spectroscopic survey mission designed to map the global history and probe the causes of star formation and evolution in galaxies ${ }^{1,2}$. Currently GALEX is set for launch in the fall of 2002. The optical design relies on large format (65mm diameter; 4096x4096 pixel) sealed tube microchannel plate (MCP) detectors with cross delay anode readouts ${ }^{3,4}$. The detector tubes have been developed and built by the Experimental Astrophysics Group at the University of California, Berkeley in collaboration with members of the Space Astrophysics Laboratory at Caltech.

GALEX requires two sealed tube detectors, (an NUV and an FUV), to achieve its desired wavelength coverage. The detectors are almost identical except for the window material and photocathode chosen for the spectral bandpass. In addition to the two flight tubes, one spare tube for channel has been delivered to JPL/Caltech. The FUV tube has a $\mathrm{MgF}_{2}$ window and a CsI photocathode deposited on the MCP forming a bandpass from 115 to $180 \mathrm{~nm}$. The NUV tube has a bandpass from 165 to $300 \mathrm{~nm}$ produced by a fused silica window with a CsTe photocathode proximity-focussed to the MCP. Figure 1 shows a diagram of the sealed tube detectors and Figure 2 is a photo of the NUV flight tube.

The photons pass through the entrance window and interact with a photocathode, resulting in photoelectron emission. The MCP then multiplies the charge through an electron avalanche to a total gain of about $10^{7}$ electrons. This charge cloud then drifts about $6 \mathrm{~mm}$ to the cross delay line (XDL) anode. The charge is divided by the two delay line electrode sets so that $\mathrm{X}$ and $\mathrm{Y}$ event positions can be determined from the differences in signal arrival time at the two ends of the anode. The overall delay line detector scheme is similar to those we have built and successfully flown on the $\mathrm{SOHO}^{5}$ and $\mathrm{IMAGE}^{6}$ missions. The differences are that this delay line is much larger and the detectors are in sealed tubes rather than open faced. 
Signals from the anode are processed by two identical separate electronic chains, one for the $\mathrm{X}$ position and the other for the Y position. The position encoding electronics begins with a fast timing amplifier which are mounted on the rear of the detector (see Figure 1) to reduce noise. The amplifier signals are then routed to the time to digital converter (TDC). Constant fraction discriminators are used to produce timing signals for each end of the delay. The time difference between start and stop signals for each axis are converted to digital positions via a coarse/fine system. A counter is used to determine the number of clock cycles between the start and stop signals to give the 3 most significant bits of the coordinate. Then start and stop interpolators measured the fine position values. The counter, fine position values, and charge amplitude are then sent to the instrument via an interface box. The positions are then determined on ground from the counter and fine position values.

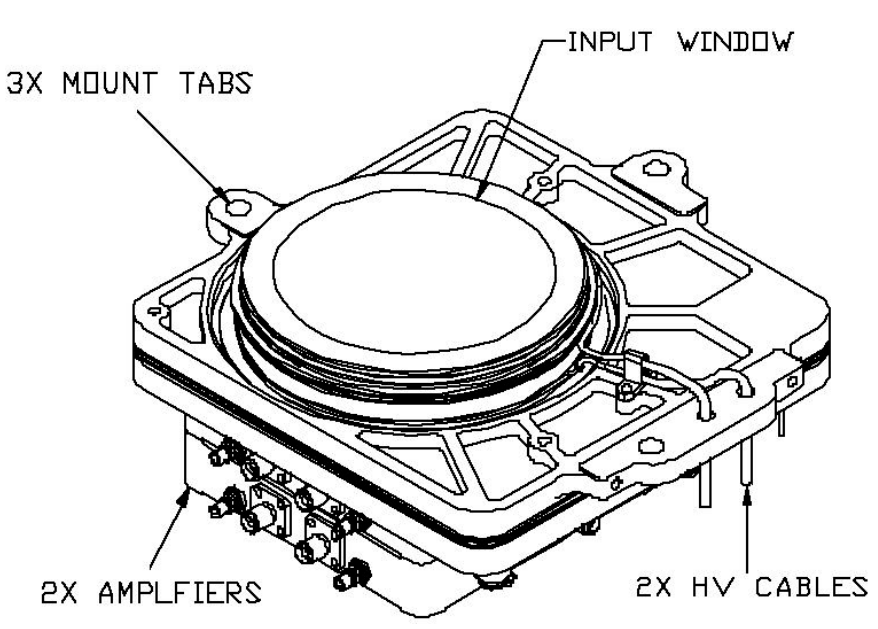

Figure 1 Diagram of GALEX sealed tube detector

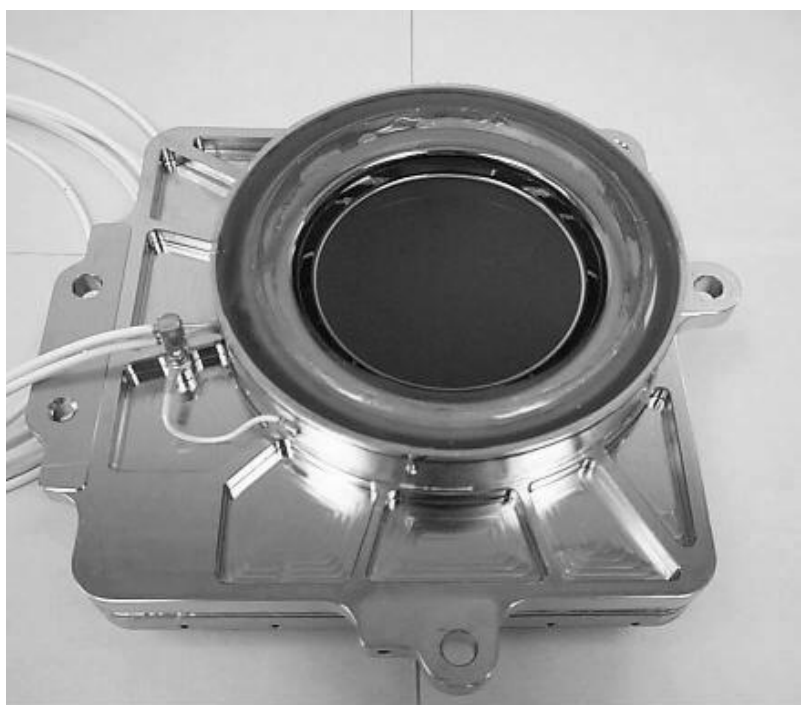

Figure 2 Photograph of the NUV detector

\section{CALIBRATION PLAN}

On a standard MCP detector much of the calibration can be done as the detector is built up. On a sealed tube though, many of the characteristics (QE, resolution, background, etc.) change once the tube is sealed. In addition, on GALEX the flight electronics were not delivered until late in the program. Therefore most of the detector calibration had to wait until the tube was completed. Also, once the tubes are sealed, nothing can come in contact with the MCP, so the pinhole masks ordinarily used in the linearity and resolution measurements can only be used before the tube is processed. Final linearity and resolution tests were performed at the instrument level where an elaborate calibration system provided a full-field grid of pinhole images.

The detector development process can be summarized as follows:

- Choose MCPs and Anodes

- Assemble detector in tube before weld

- Test spatial linearity and resolution using masks in contact with front MCP surface with rack electronics

- Coat FUV photocathode and test quantum efficiency (QE) (not done with NUV tube).

- Weld and process tube

- Calibrate quantum efficiency

- Lifetest the detector to $10-20 \%$ of mission counts.

- Finish with standard flat field, gain-voltage and background tests, which become benchmarks for standard functional tests.

- Calibrate linearity and resolution at Instrument level. 
A subset of the results of these calibrations for the flight GALEX detectors is presented below. Both detectors have been integrated into the instrument at JPL/Caltech and have accumulated over 1000 hours of environmental testing. The instrument was integrated into the spacecraft in the fall of 2001, and a majority of the spacecraft testing has been completed in preparation for launch in fall 2002.

\section{CALIBRATION RESULTS}

\subsection{Gain Voltage and Pulse Height Distribution}

The detectors were operated at several voltages to optimize their performance. The gain versus voltage curves for both tubes are shown in Figure 3. They are very similar except the FUV requires about 1000 volts more for the same gain, partially due to a larger window-MCP voltage and partially due to a lower inherent MCP gain in the FUV MCP stack. The tubes are typically operated at a gain between $10^{7}$ and $2 \times 10^{7}$ to optimize the imaging performance. The pulse height distributions are also shown in Figure 4. Both tubes are similar with a FWHM of about $95 \%$ for the NUV and $140 \%$ for the FUV tube. The large FWHM is mostly caused by gain variations across the large area of the MCP.

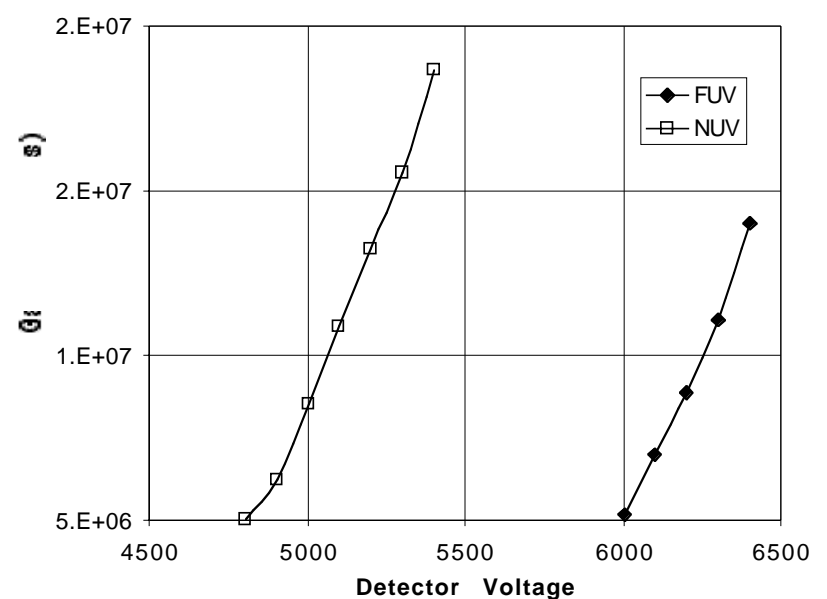

Figure 3 Gain versus applied voltage for the FUV and NUV tubes.

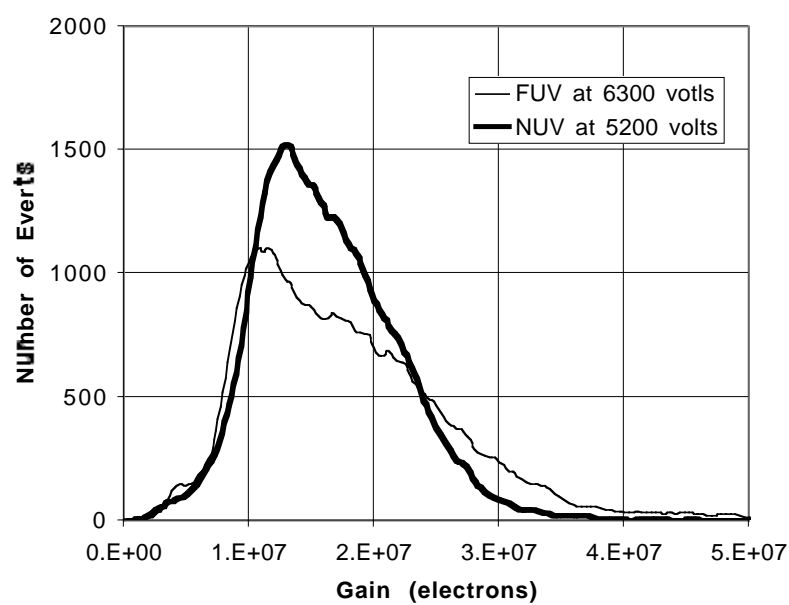

Figure 4 Pulse height distribution for the FUV and NUV tubes. The large FWHM is due to gain variations across the large area of the tubes.

\subsection{Quantum Efficiency}

For detector-level QE testing, the tubes were installed on a 2 axis manipulator in a vacuum calibration chamber at the Space Sciences Laboratory. The manipulator allowed horizontal and vertical motion. An FUV (or EUV) beam from a hollow cathode discharge source was sent through a monochromator and then through a pinhole into the tank. This beam could be measured by either a $\mathrm{MgF}_{2}$ windowed FUV diode $(\lambda<2500 \AA)$ or phototube $(\lambda>2500 \AA)$. The intensity of the input beam was determined from the current in the FUV diode or the count rate in the phototube. The QE is then the ratio of the count rate in the detector to the intensity of the input beam Figure 5 shows the QE of the FUV tube and Figure 6 shows the QE of the NUV tube including the transmission of the windows. In addition, the relative QE was measured at several positions on the detector.

The high GALEX quantum efficiency (QE) specification was very difficult to meet. The QE of the FUV cathode degraded at long wavelengths due to the bake and UV scrubbing process needed to seal the tube. There was not enough time to develop a technique of depositing an opaque CsTe cathode on the MCP in the NUV tube. In principal, this would have yielded higher QE and better resolution. However, the QE of the NUV tubes (see Figure 6) is similar to other MCPbased detectors on orbit, such as the STIS MAMA on board the Hubble Space Telescope, whereas the FUV QE , Figure 5 , is somewhat lower than the STIS MAMA performance. 


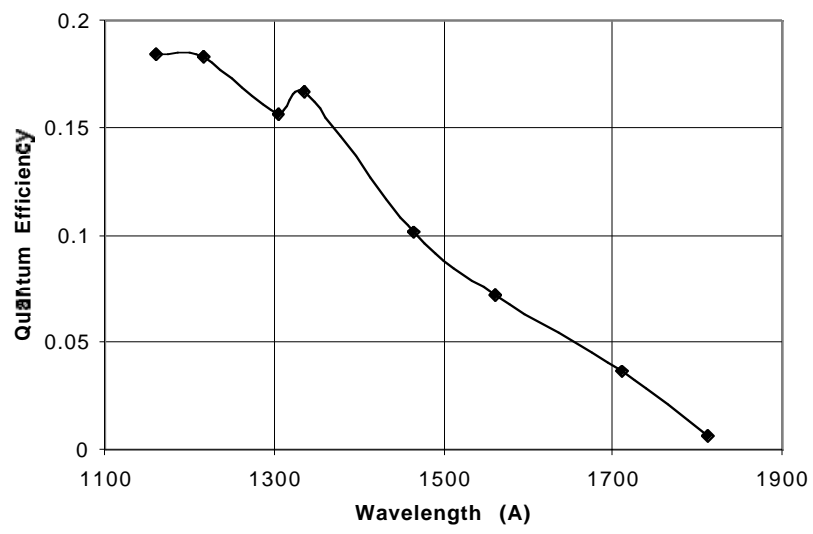

Figure 5 Quantum Efficiency of the FUV tube, including window transmission.

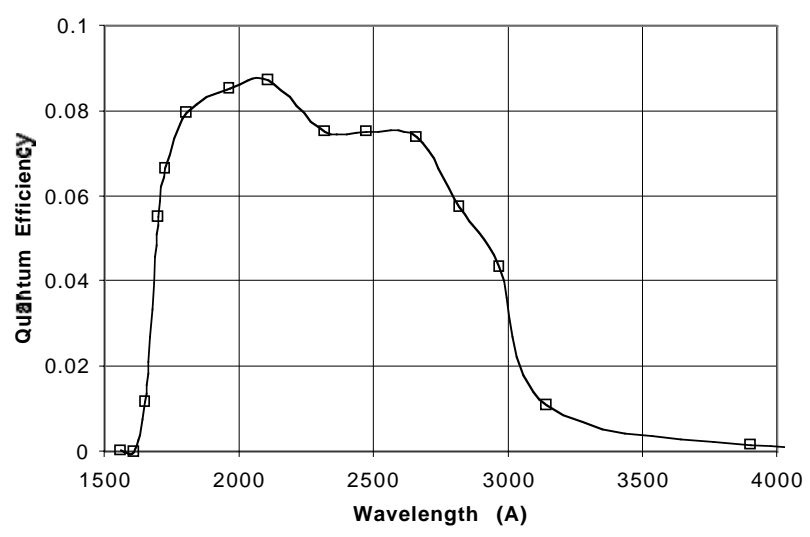

Figure 6 Quantum Efficiency of the NUV tube, including window transmission.

\subsection{Background}

After the tube was sealed the background (dark) rate was measured. Figure 7 shows background image the FUV tube and Figure 8 shows the background image for the NUV tubes just before delivery. In the FUV image there are many hotspots and an area of field emission to the lower left of the image. The NUV image has many fewer hotspots and two areas where the background is enhanced near the edge on the upper left and lower right.

The FUV tube was operated at 6300 volts. The background is dominated by hotspots. The rate of the hotspots is about 400 counts/second. The diffuse rate is about 0.5 counts $/ \mathrm{sec} / \mathrm{cm}^{2}$. In addition, there is an area near the edge with about 7 counts/second of field emission. In the 1.5 years since delivery, the hotspots have quieted down to about 20 counts/second, the diffuse rate has quieted down to about 0.3 counts $/ \mathrm{sec} / \mathrm{cm}^{2}$, and the field emission area has remained about the same.

The NUV tube was operated at 5200 volts. The background was probably dominated by room light. Even though the CsTe cathode is solar blind, it still is responsive to room light. The rate of hotspots was about 100 counts/second. The diffuse rate was about 2.5 counts $/ \mathrm{second} / \mathrm{cm}^{2}$. During the 2 years since delivery, the hotspots have remained about the same, but the diffuse rate is down to about 0.9 counts $/ \mathrm{sec} / \mathrm{cm}^{2}$.

Figure 9 shows the pulse height distribution of the background for the NUV tube. The dark line is the pulse height distribution of all of the background (including hot spots), and the light line is the distribution for just the diffuse events(hotspots removed). By comparing to Figure 4, the diffuse events have a pulse height distribution very near that of photons, and the total which is dominated by hotspots has a much lower gain. The FUV curves are very similar, except that the hotspots dominate the diffuse, so they have not been shown.

\subsection{Life test}

The tubes were put through a life test to screen out ones that would potentially fail in the future. The final tubes were placed in an oven to keep them at $30^{\circ} \mathrm{C}$ (the highest flight operational temperature). An UV light source was the used to illuminate the tube. The tube was run at full gain until the number of counts accumulated was about $10 \%$ of the counts expected during a GALEX mission. The NUV tube ran at 70,000 counts/second for 8.7 days for a total of $5.3 \times 10^{10}$ counts. The FUV tube ran at 41,000 counts/second for 2 days for a total of $7.2 \times 10^{9}$ counts. The characteristics of the tube (flat field, gain, and background) were monitored before, during and after the life test. All the tubes performed the same or better after the life test. 


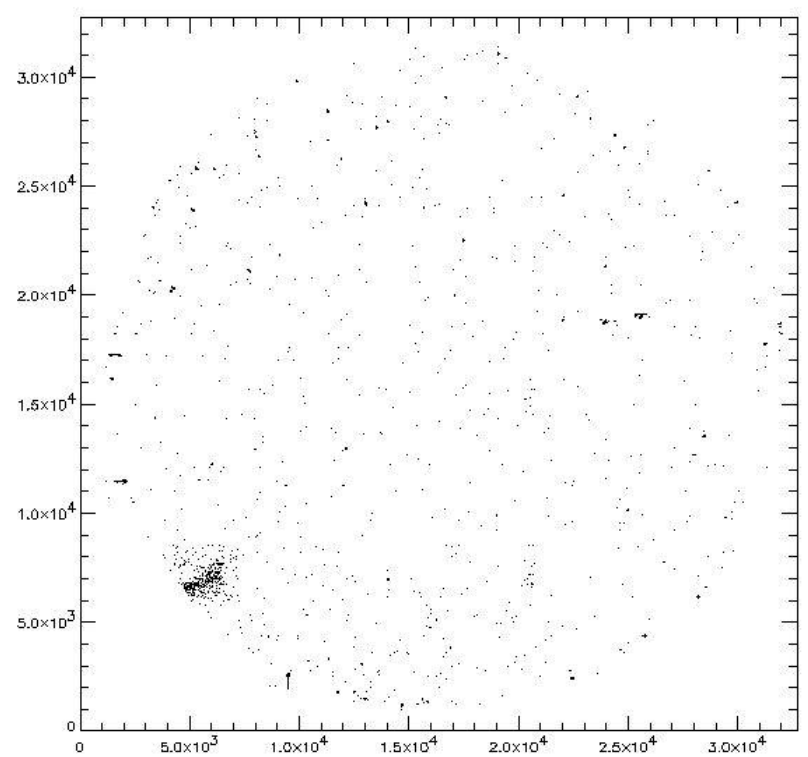

Figure 7 Pre delivery background image of the FUV tube at 6300 volts.

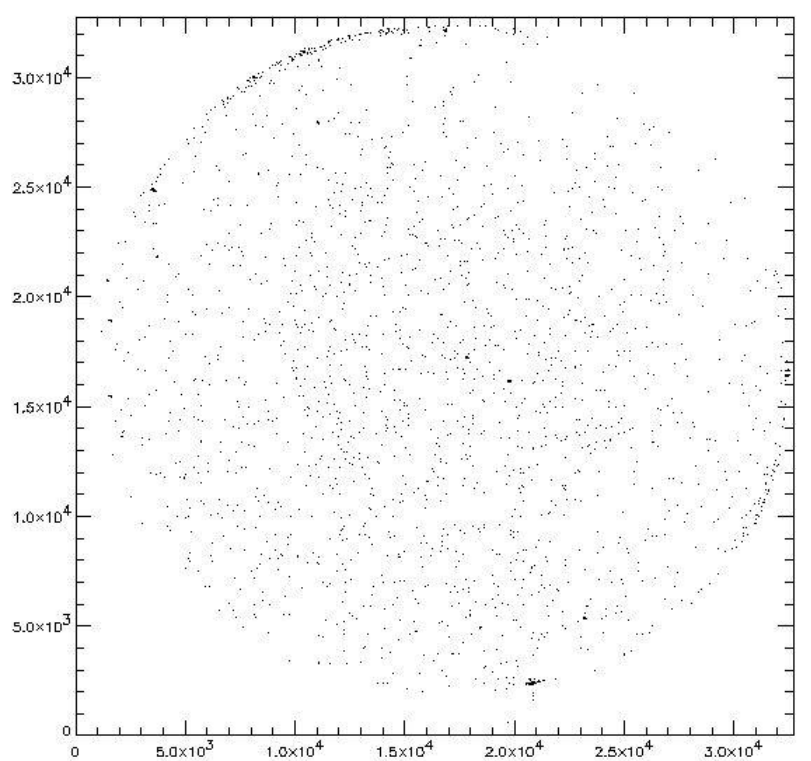

Figure 8 Pre delivery background image of the NUV tube at 5200 volts.

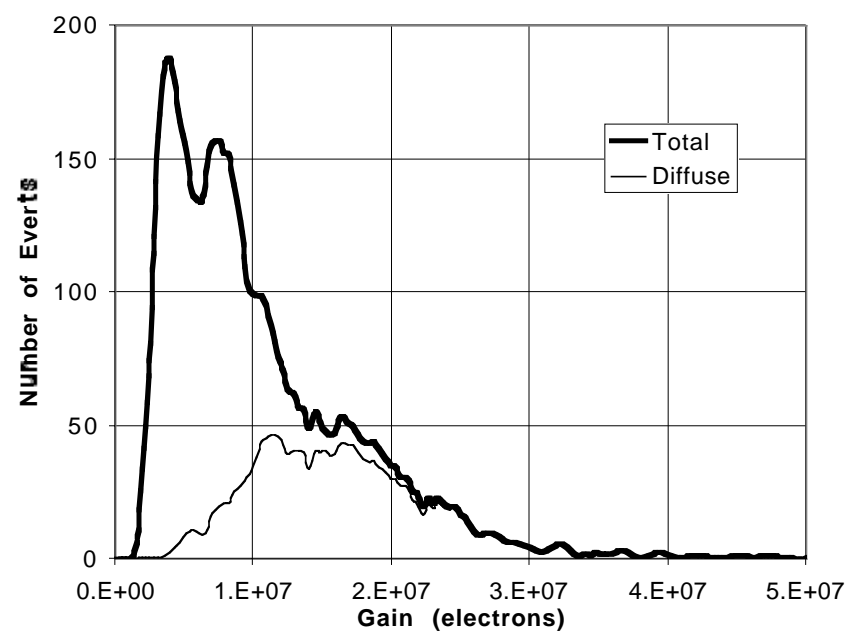

Figure 9 NUV background Pulse height distribution. Dark curve is all the background (including hotspots), and the light curve is just the diffuse background (no hotspots).

\subsection{Flat fields}

The flat fields measure the uniformity of the response of the tube to photons. They also can be used to measure the uniformity of the gain of the tube. An ultraviolet (UV) source was used to place light onto the tube face and rack electronics were used to acquire the data. Figures 10 and 11 show the flat field images for both the FUV and NUV tubes taken before delivery. The FUV image was a 20 million event integration taken at 6300 volts. The stripes in the image are caused by metal QE-enhancing wires on the window, which detect optical light that the photocathode does not. These are reduced by a huge amount when just in band light is used. The NUV image was a 10 million event integration taken at 5200 volts. The illumination of the NUV tube was difficult to make uniform due to it's room light sensitivity. 


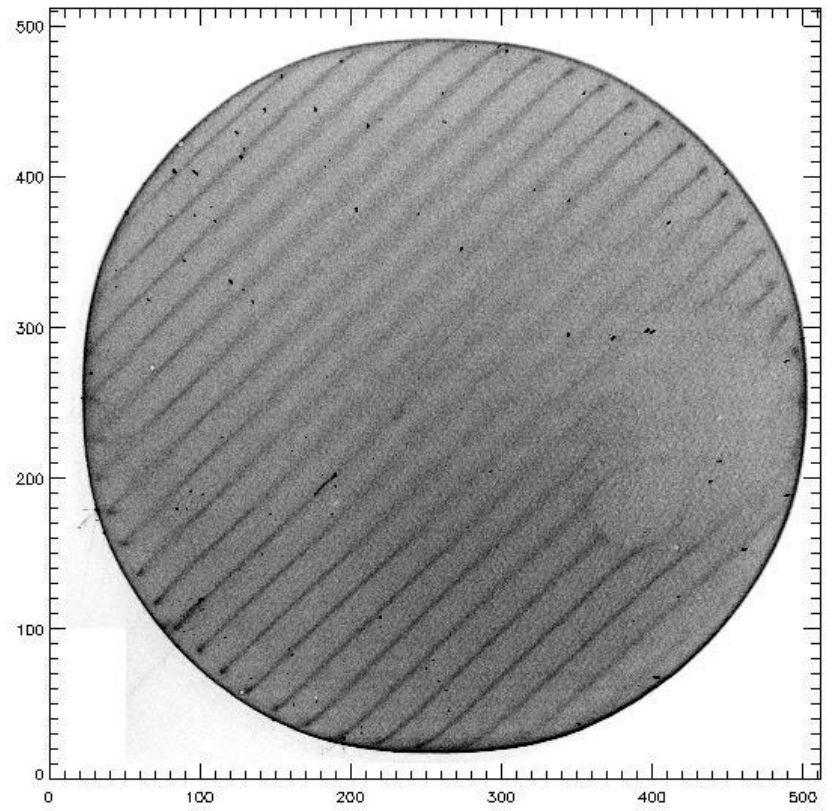

Figure 10 FUV flat field image taken at 6300 volts. The stripes are caused by metal wires on the window, which are more sensitive to optical light than the photocathode.

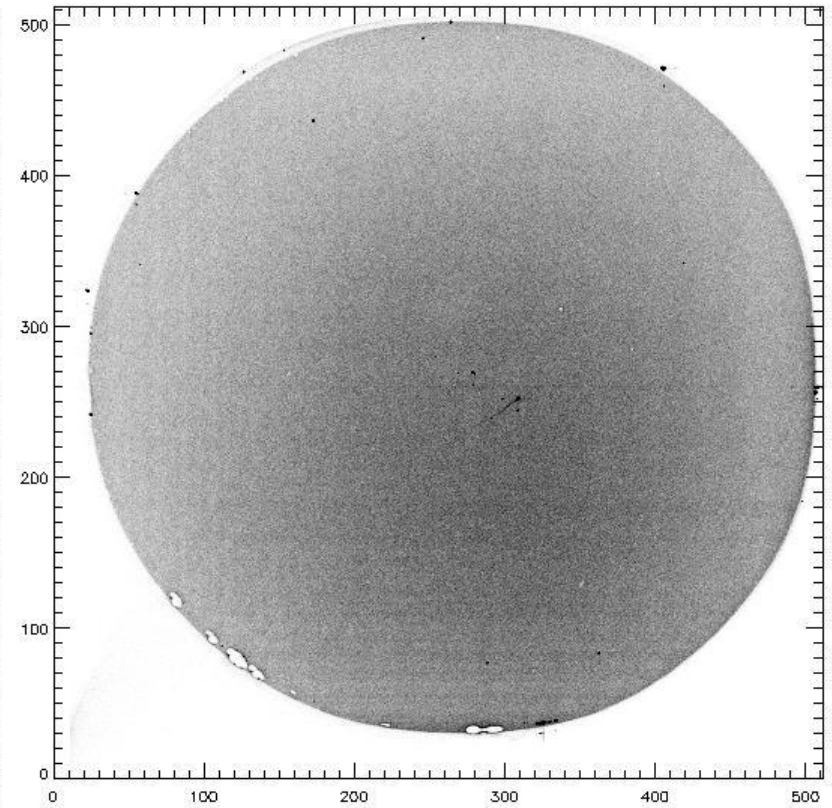

Figure 11 NUV flat field image taken at 5200 volts.

\subsection{Spatial Linearity and Resolution}

The resolution and linearity of the tubes could only be measured at the Space Sciences Laboratory with rack electronics before the tube was sealed. A mask with $10 \mu \mathrm{m}$ diameter pinholes spaced on a $1 \mathrm{~mm} \times 1 \mathrm{~mm}$ grid was placed in contact with the top MCP. UV light was used to illuminate the MCP through the pinholes. Figures 12 and 13 show the

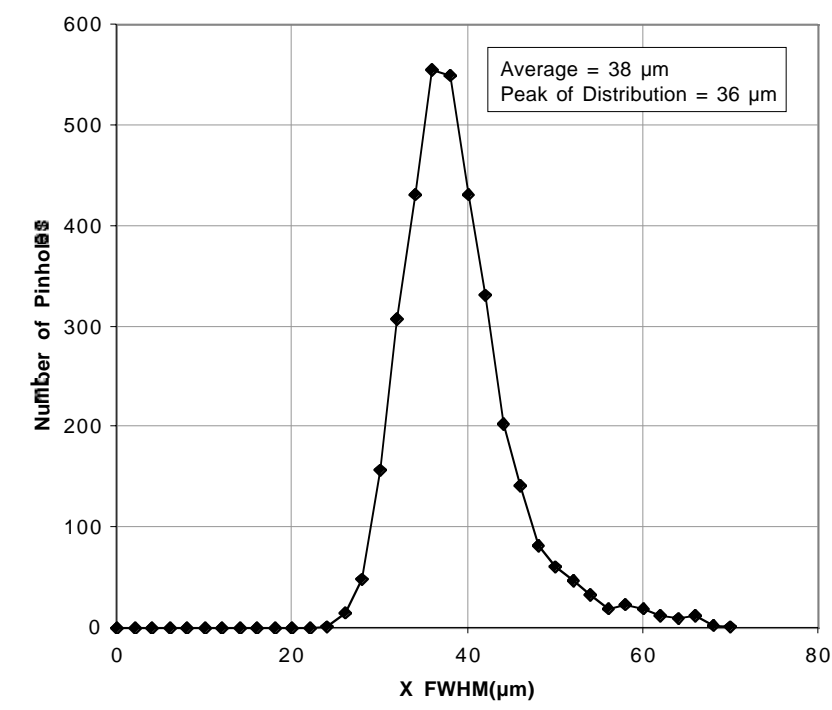

Figure 12 FUV resolution histogram (before seal) of $X$ FWHM. Number of pinholes with a given resolution. Average is $38 \mu \mathrm{m}$ and the peak of the distribution is $36 \mu \mathrm{m}$.

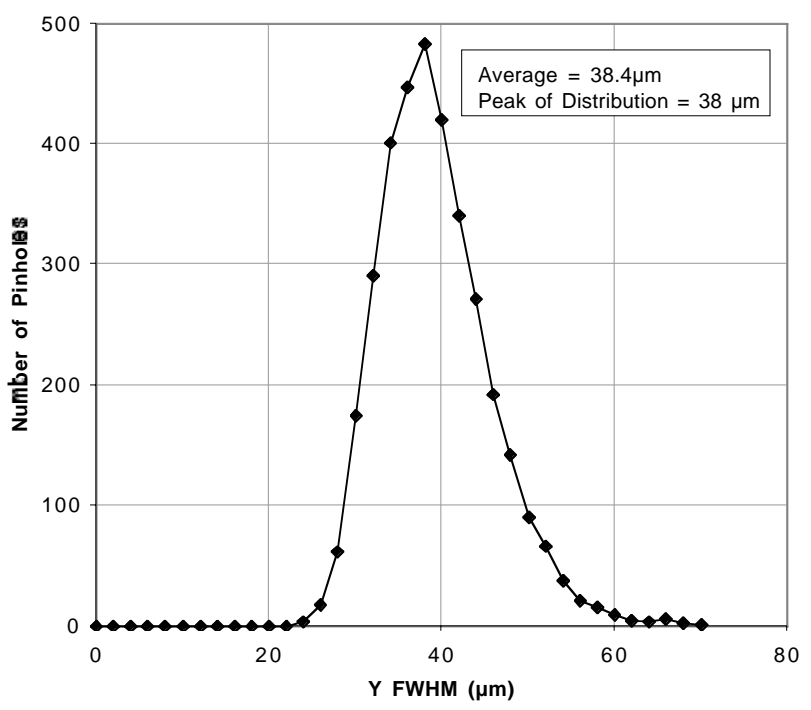

Figure 13 FUV resolution histogram (before seal) of Y FWHM. Number of pinholes with a given resolution. Average is $38.4 \mu \mathrm{m}$ and the peak of the distribution is $38 \mu \mathrm{m}$. 
distribution of resolutions for the FUV tube run at a gain of $1.5 \times 10^{7}$. The average full width at half maximum (FWHM) is about $38 \mu \mathrm{m}$, given the size of the pinholes, then actual detector resolution with rack electronics is about $36 \mu \mathrm{m}$. The resolution of the NUV tube before seal is not shown. It's resolution will be strongly modified by the proximity focus of the photocathode to MCP in the final tube.

The linearity of the instrument was measured at JPL. A mask with $200 \mu \mathrm{m}$ diameter pinholes was illuminated and placed at the focus of a duplicate telescope. The collimated light from the duplicate telescope was directed to the instrument. The instrument formed images of the pinhole mask on the detector. The data was analyzed to produce a correction map. Figure 14 shows the linearity image used for the FUV analysis. The magnitude of the corrections is shown in Figure 15. Most of the non linearity is probably caused by the flight electronics not the tube itself. The position of the spot can be corrected to about 15 microns ( 1 arc-second) for about 3/4 of the field of view.

The resolution of the instrument was measured in a similar manor but using a mask with $20 \mu \mathrm{m}$ pinholes. The resolution includes the resolution of both of the telescopes and the detector contribution. This means that it is an upper limit to the detector resolution. The measured resolution for the FUV channel was about $105 \mu \mathrm{m} 80 \%$ encircled energy (EE) diameter. The collimator and telescope add about $41 \mu \mathrm{m}$ due to astigmatism and the pinholes add about $20 \mu \mathrm{m}$. Therefore the derived resolution of the FUV tube with flight electronics is about $80 \mu \mathrm{m} 80 \%$ EE or about $53 \mu \mathrm{m}$ FWHM. The measured resolution of the NUV channel was $135 \mu \mathrm{m} 80 \%$ EE diameter. Therefore the derived resolution of the NUV tube with flight electronics is about $117 \mu \mathrm{m} 80 \% \mathrm{EE}$ diameter of about $78 \mu \mathrm{m}$ FWHM. The NUV tube is probably worse due to the blur due to the proximity focus of the photocathode to the MCP. The resolution is significantly worse than the data from Figures 12 and 13. This is probably due to the flight electronics and gain differences after the tube was sealed.

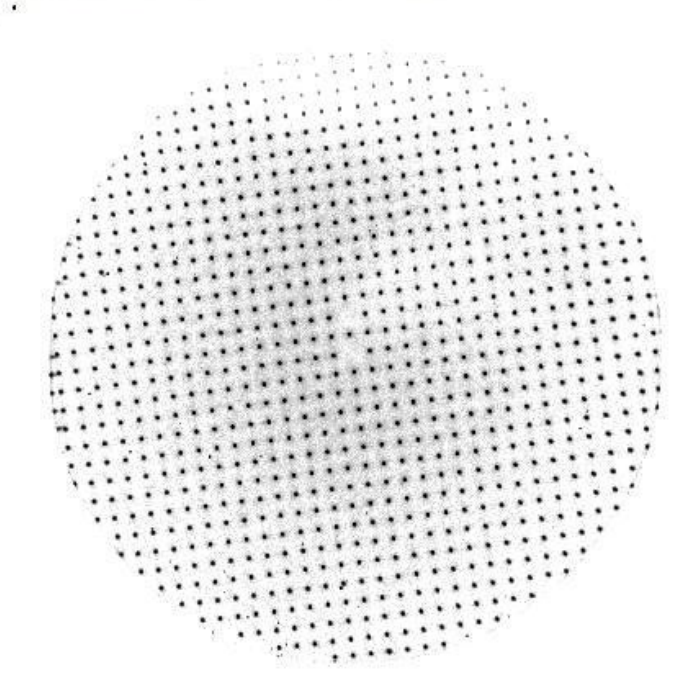

Figure 14 Image used for FUV linearity.

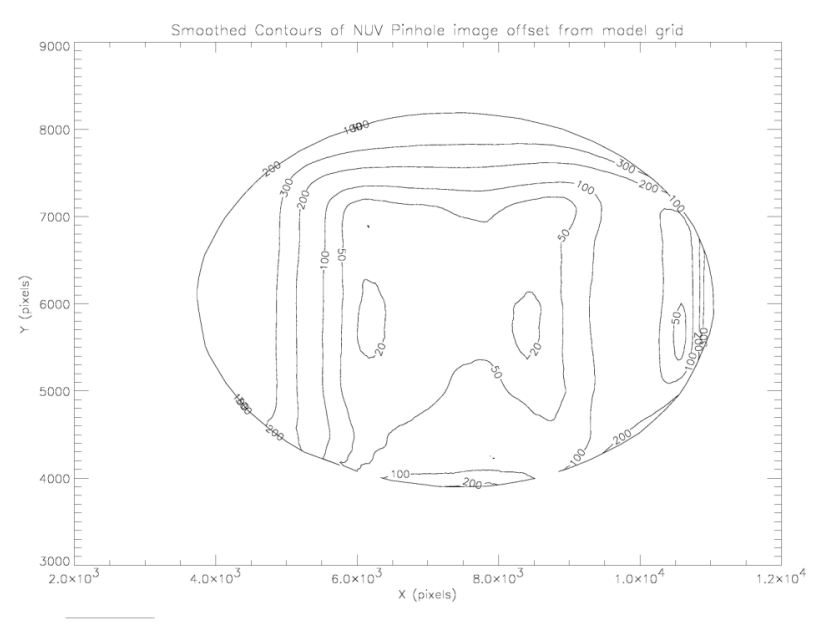

Figure 15 Linearity Contours for FUV channel in $\mu \mathrm{m}$.

\section{ACKNOWLEDGEMENTS}

The authors would like to thank the GALEX teams at Caltech and JPL. We would also like to thank Darrel Doliber and Joseph Stock for their large contribution in the construction and characterization of the tubes. This work was supported by Caltech under contract \#PC251341 and GSFC grant \# NAG5-7615.

\section{REFERENCES}

1. C. D. Martin, L. Bianchi, J. Donas, T. Heckman, B. Madore, R. Malina, B. Millard, P. Friedman, M. Rich, D. Schiminovich, O. H. W. Siegmund, and A. Szalay, "The Galaxy Evolution Explorer", Ultraviolet-Optical Space 
Astronomy Beyond HST, ASP Conference Series 164, J. A. Morse, J. M. Shull, \& A. L. Kinney. ISBN: 1-88673385-6, p. 182, 1999.

2. C. D. Martin, et al., "The Galaxy Evolution Explorer", Proc. SPIE, 4854, 2002. (this conference)

3. O. H. Siegmund, P. N. Jelinsky, S. R. Jelinsky, J. M. Stock, J. S. Hull, D. L. Doliber, J. Zaninovich, A. S. Tremsin, K. E. Kromer, "High-resolution cross delay line detectors for the GALEX mission", Proc. SPIE, 3765, pp. 429-440, 1999.

4. D. L. Doliber, J. M. Stock, S. R. Jelinsky, J. Malloy, P. N. Jelinsky, O. H. Siegmund, J. S. Hull, " Development challenges for the GALEX UV sealed tube detectors", Proc. SPIE, 4013, pp. 402-410, 2000.

5. O. H. Siegmund, J. M. Stock, D. R. Marsh, M. A. Gummin, R. Raffanti, J. Hull, Jeffrey, G. A. Gaines, B. Y. Welsh, B. Donakowski, P. N. Jelinsky, T. Sasseen, J. L. Tom, B. Higgins, T. Magoncelli, J. W. HamiltonS. J. Battel, A. I. Poland, M. D. Jhabvala, K. Sizemore, J. Shannon, "Delay Line Detectors for the UVCS and SUMER Instruments on the SOHO Satellite", Proc. SPIE, 2280, pp. 89-100, 1994.

6. J. M. Stock, O. H. Siegmund, J. S. Hull, K. E. Kromer, S. R. Jelinsky, H. D. Heetderks, M. L. Lampton, S. B. Mende, "Cross-delay-line microchannel plate detectors for the Spectrographic Imager on the IMAGE satellite" Proc. SPIE, 3445, 407, 1998. 